\title{
Why a Good Catalyst Can Turn Out Detrimental to Good Polymerization
}

\author{
Mikel Abadia, ${ }^{\dagger} \ddagger$ Jens Brede, ${ }^{\dagger}$ Alberto Verdini, " Luca Floreano, "Pawel Nita, ${ }^{\dagger}$, \\ Dimas G. de Oteyza, ${ }^{\dagger, \S, \|}$ Jose Enrique Ortega, ${ }^{\dagger, \S, \perp}$ Martina Corso, ${ }^{\dagger, \S}$ and Celia \\ Rogero*,, §
}

$\dagger$ †aterials Physics Center (CSIC-UPV/EHU), E-20018 San Sebastian, Spain

$\ddagger$ Present address: Eureka! Zientzia Museoa, E-20009 San Sebastian, Spain

ฯ CNR-IOM, Laboratorio Nazionale TASC, I-34149 Triestre, Italy

$\S$ Donostia International Phsics Center DIPC, E-20018 San Sebastian, Spain

||Ikerbasque, Basque Foundation for Science, E-48011 Bilbao, Spain

$\perp$ Departamento Fisica Aplicada I (UPV/EHU), E-20018 San Sebastian, Spain

E-mail: celia.rogero@csic.es 


\section{c-Au(111) Crystal}

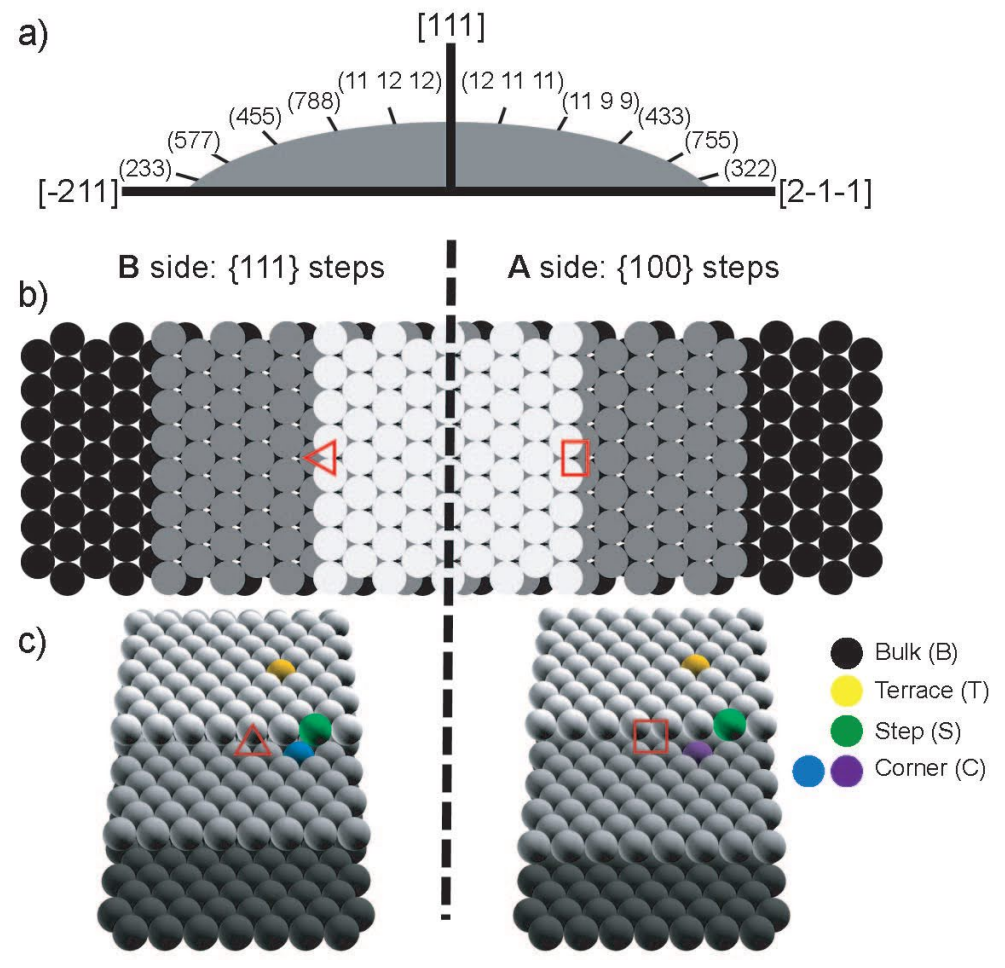

Figure S1: Three perspectives of the c-Au(111) sample, a cylindrical section of a Au single crystal, with cylinder axis along the [1-10] direction and the center of the curved surface along the [111] direction: side view (a)with some high symmetry directions indicated; top view (b) where B (red triangle) and A (red square) type of steps are distinguished; front view (c)with the different coordination highlighted. Non-equivalent surface atoms, i.e. bulk, terrace, step and corner atoms, are remarked in different colors. With such sample, Au(111) vicinal surfaces of increasing vicinal $(\alpha)$ angle, featuring $\{100\}$ (A-type) and $\{111\}$ (B-type) microfacets are found by moving away from the center, up to $\alpha \pm 15^{\circ}$. The surface atoms have different total coordination. The $B$ atom has the highest coordination with 12 atoms around, the $T$ atom is surrounded by another 9 atoms and finally, the $S$ atom is the least coordinated one with 7 atoms around. Only the coordination of the corner $(C)$ atom is different depending on the step type. The corner atom has a coordination of 10 or 11 in $\mathrm{A}$ or B-steps, respectively. 


\section{Coordination-dependent core-level shift measured on dif-}

\section{ferent Au surfaces}

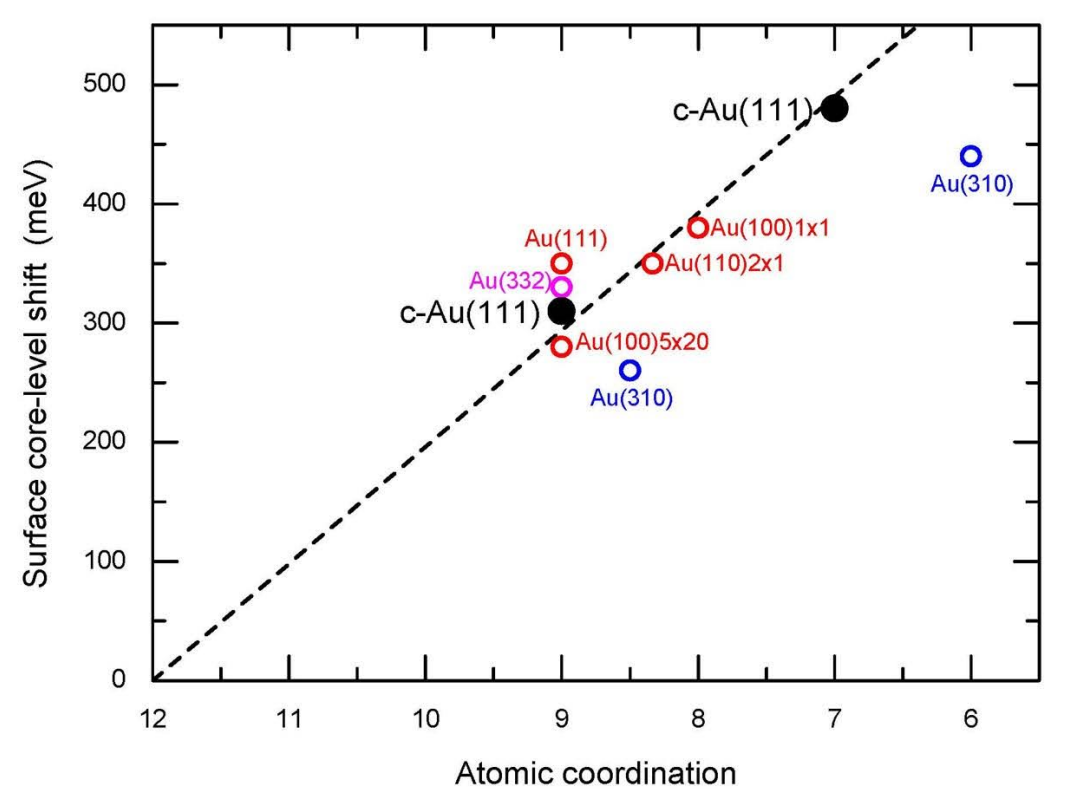

Figure S2: Core-level shifts measured for surface atoms in a variety of Au crystal surfaces as a function of the atomic coordination of Au atoms at different surfaces(values referred to bulk component). There is a linear variation of the core-level shift as a function of the atomic coordination of $\mathrm{Au}$ atoms at different surfaces. Data for $\mathrm{Au}(111), \mathrm{Au}(100) 1 \times 1$, $\mathrm{Au}(110) 2 \times 1$, and $\mathrm{Au}(100) 5 \times 20$ are taken from Ref. 1, and for $\mathrm{Au}(223)$ and $\mathrm{Au}(310)$ from Refs. 2 and 3, respectively. The big black dots correspond to the values determined from the analysis of Figure 2 in the main text. The dotted line represents a linear fit to data points intersecting the origin of coordinates (bulk line) 


\section{LEED evolution at different surfaces of the crystal}

(335)

$0.95 \mathrm{~nm}$ terrace

1 PPP chain per terrace

(223)

$1.2 \mathrm{~nm}$ terrace, $3.5 \mathrm{~nm}$ faceting 3 PPP chains per facet

(779)

$1.9 \mathrm{~nm}$ terrace 1 PPP chain domain

111

3 PPP chain domains
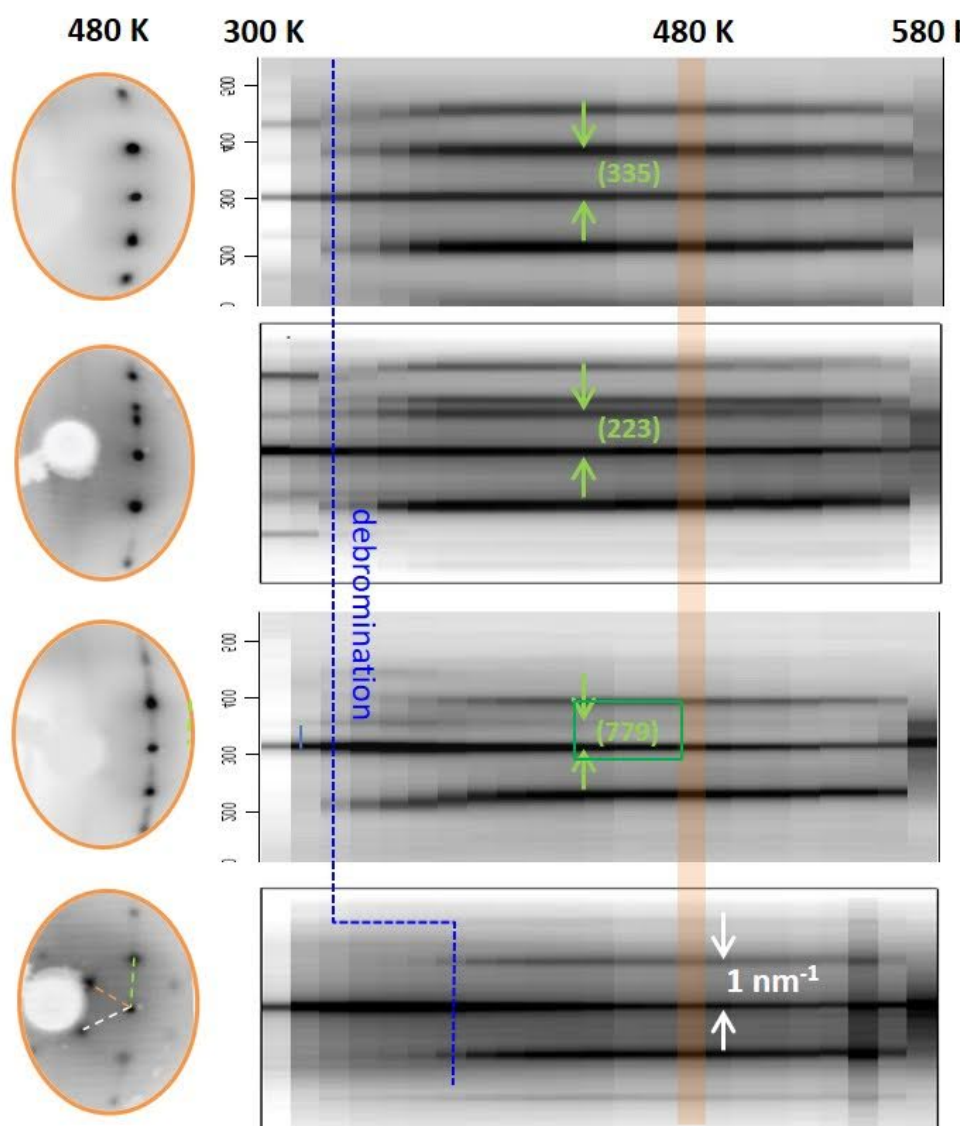

Figure S3: Low Energy Electron Diffraction patterns at four different sample positions of the curved c-Au(111)acquired after annealing the DBTP covered surfaces at 480K. The intensity variation of the spots as a function of the temperature is also included. The emergence of characteristic PPP beam and the facetings are indicated. 


\section{ARPES before and after polymerization of DBTP}

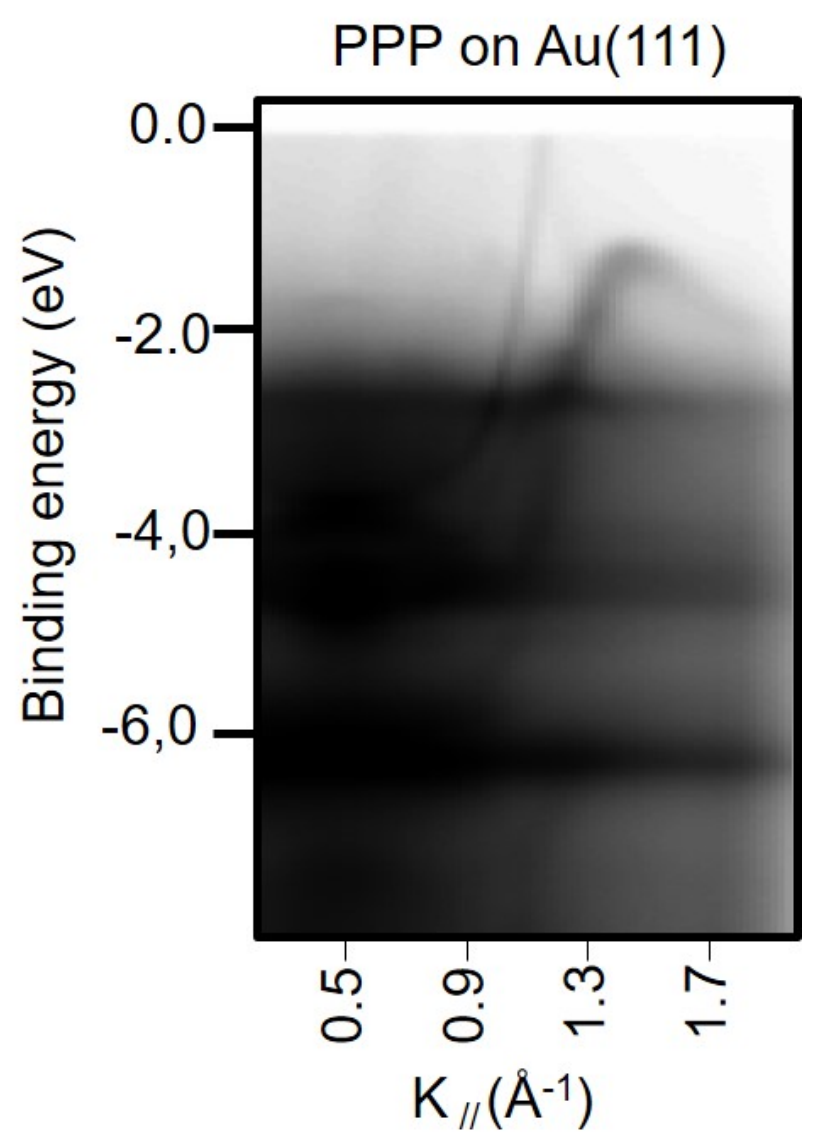

Figure S4: Complete ARPES spectra of PPP at Au(111), center of the c-Au crystal.

\section{References}

(1) Heimann, P.; van der Veen, J.; Eastman, D. Structure-dependent surface core level shifts for the $\mathrm{Au}(111),(100)$, and (110) surfaces. Solid State Communications 1981, 38, 595 $-598$.

(2) Prieto, M. J.; Carbonio, E. A.; Landers, R.; de Siervo, A. Promotion Effect of Platinum on Gold's Reactivity: A High-Resolution Photoelectron Spectroscopy Study. The Journal of Physical Chemistry C 2016, 120, 10227-10236. 
(3) Weststrate, C.; Lundgren, E.; Andersen, J.; Rienks, E.; Gluhoi, A.; Bakker, J.; Groot, I.; Nieuwenhuys, B. $\{\mathrm{CO}\}$ adsorption on $\mathrm{Au}(3 \mathrm{l}$ 0) and $\mathrm{Au}(32$ 1): 6-Fold coordinated gold atoms. Surface Science 2009, 603, 2152 - 2157. 